\title{
Sequential Color Recapture and Dynamic Filtering: A Method of Scrolling Color
}

\author{
D. Scott Dewald, Steven M. Penn, and Michael Davis \\ Texas Instruments Incorporated, DLP ${ }^{\mathrm{TM}}$ Products Division, Plano TX, USA \\ Permission for publication, courtesy of Society for Information Display
}

\begin{abstract}
Scrolling color has long been a goal of the projector industry, as it enables the most efficient use of light in a single panel display. Current methods of implementing scrolling color use the techniques of splitting the light into primary colors, and manipulating that light on the modulator. The authors present the techniques of dynamic filtering and sequential color recapture (SCR) to achieve the same result with no moving components other than a color wheel, showing that the efficiency of 3 modulator systems can be approached with one modulator. Analysis of the technique applied to DLP ${ }^{T M}$ projection displays, and results of prototype projection systems using the techniques, will be presented.
\end{abstract}

\section{Introduction}

Creating a full color display with a single modulator has been a goal of the projector design community for some time. Currently, the accepted method used is sequential color, or "field sequential color" $[1,2]$. In this method a sufficiently fast modulator (DMD or LCD) creates three or more modulated images in primary colors per video frame, which are switched sufficiently fast to create a full color image in the human visual system. This is the method used in many 1-chip $\mathrm{DLP}^{\mathrm{TM}}$ projectors in the market today.

Another method of full color display using a single modulator is a scrolling color method, several types of which are described in the literature [3, 4]. This method has several advantages, the first being that all colors are present on the modulator at the same time, so the waste of light caused by field sequential color is avoided. The second advantage is the reduction of "color separation artifacts", which are caused by quick eye movements or a fast changing scene when viewed on a field sequential color display. This is caused by successive colored fields of the image being sufficiently displaced on the retina to yield a "fringe" of colored light at a white-black boundary for instance.

Scrolling color optics in the literature use numerous optical surfaces and moving optical components to manipulate the color bands on the modulator. This is quite complex mechanically, and has been shown to be much less efficient than theory predicts. The authors show sequential color recapture (SCR) as a solution to the scrolling color problem with a minimum of optical components.

\section{SCR Components}

SCR was made possible by advances in dichroic and metal thinfilm technology, the most important of these being the photolithographic patterning of dichroic coatings. Similar to today's 1-chip DLP ${ }^{\mathrm{TM}}$ projectors, a rod-type integrator and color wheel are used with modifications as described below.

\subsection{SCR Integrator}

Integrators for SCR are similar to standard rod-type integrators with the addition of an "entrance aperture", formed by mirroring the input end of the integrator, with the exception of a circular transparent area of approximately $1 / 3^{\text {rd }}$ the cross-sectional area of the integrator. The size of the input aperture can be adjusted to maximize the efficiency or the projector and is a function of the etendue of the lamp and the device, as will be described below. SCR integrators have been fabricated out of solid glass as well as high-reflectance "light tunnel" material. See Figure 1.

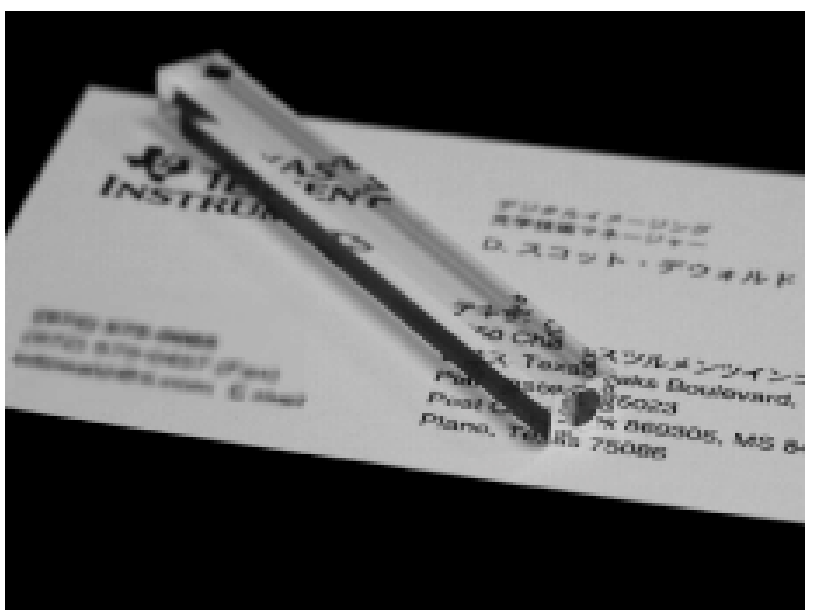

Figure 1. - SCR Integrator (solid)

\subsection{SCR Wheel}

The SCR wheel is created from RGB dichroic coatings arranged in a "spiral of Archimedes" pattern. The pattern should be sized such that one RGB pattern covers the cross-section of the output end of the integrator (Figure 2). The Archimedes spiral, defined by the equation $\mathrm{R}=\mathrm{a} \theta$, has the property that the boundary between colors moves at a constant speed in the radial direction. This causes the RGB pattern to move at nearly a constant speed over the output face of the SCR integrator described above. For best performance, transmission of the dichroic coatings should be maximized in-band, and reflection maximized out-of-band. It is also possible to include a "white segment", or a clear area which can be used to increase luminous efficiency in non-saturated images.

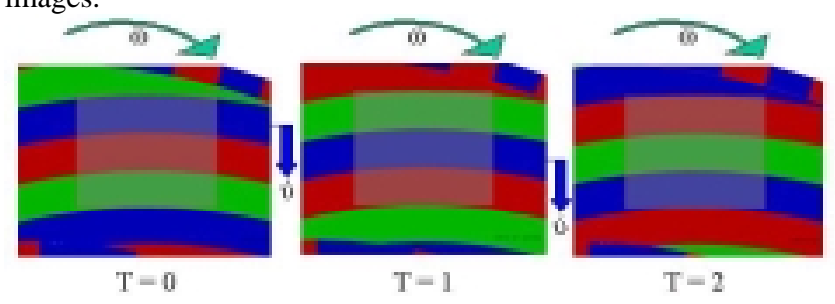




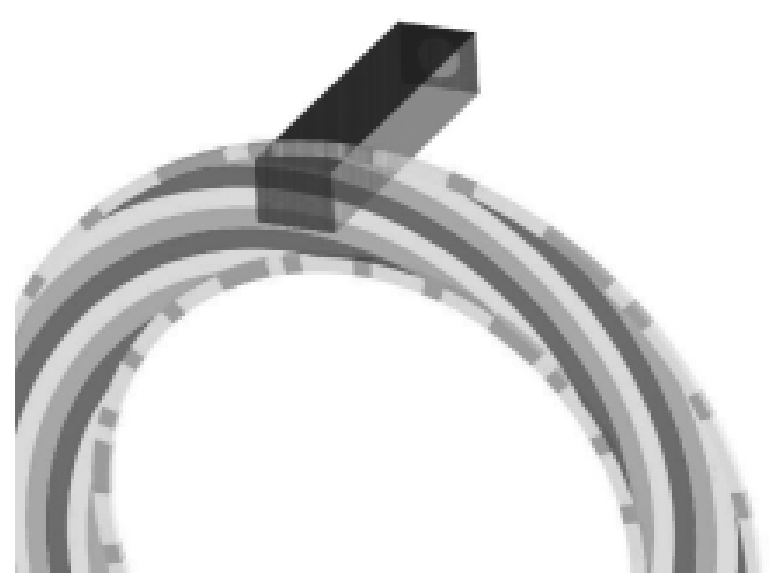

Figure 2 - SCR wheel and integrator

The spiral pattern is manufactured using a proprietary lift-off photolithographic technique that results in a very accurate patterning of the 3 dichroic coatings. The number of RGB stripes determine the speed of the wheel rotation, e.g., a wheel with many RGB sets can rotate at a slower speed for a given "scan rate" (the frequency at which a given color makes a full sweep of the modulator). A photograph of an SCR prototype wheel is seen in Figure 3.

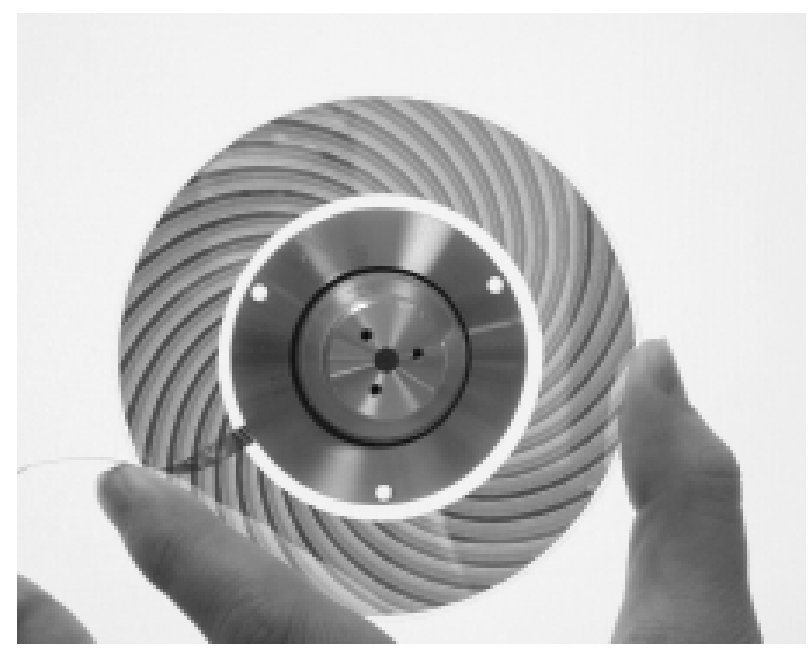

Figure 3 - SCR Wheel

The SCR process also allows smaller wheels to be used in compact projectors. Depending on the ability of the modulator to handle curved color boundaries, the wheel size may be limited by the actual motor diameter and the integrator size. The authors have investigated SCR color wheels of less than $35 \mathrm{~mm}$ diameter.

\subsection{Relay Optics}

The SCR wheel is placed such that the reflective dichroic spiral is very close to the output end of the integrator. A relay optic system then creates an image of the output end of the integrator onto the light modulator at the proper $\mathrm{f} / \#$ and magnification. As the SCR wheel turns, it can be seen that slightly curved bands of each primary color move across the modulator at a near-constant speed. With the proper formatting of data, a scrolling color image can be realized.
The importance of the integrator-wheel interface should be noted. The gap between the two components should be as small as possible, as light "leakage" can occur around the perimeter of the interface. Also, the runout of the wheel/hub/motor assembly will cause a variation of the spacing with time which can cause a visible pulsing of the image if the motor speed is below the threshold of human vision, approximately 3000RPM. Modeling of the SCR process qualitatively indicates that the total runout should be less than $.1 \mathrm{~mm}$.

\section{The SCR process \\ 3.1 Recycling of input light}

Light from a small-arc lamp is focused onto the input aperture of the integrator. The light that passes through the input aperture is homogenized by multiple reflections off the wall of the integrator. If the integrator is sufficiently long the light distribution at the output end will be fairly uniform. When the white light reaches the SCR wheel, light of a given color will transmit through the section of the wheel with the corresponding transmissive coating, while reflecting off the remaining $2 / 3^{\text {rd }}$ of the illuminated area. In other words, red light will pass through the area of the wheel covered by the red dichroic segment, but will be reflected back towards the opposite end of the integrator by the blue and green segments. This effect occurs continuously with light of all three colors.

The light that is reflected by the wheel continues to reflect off the walls of the integrator, further homogenizing the rays, until the input aperture is reached. At this point $2 / 3^{\text {rds }}$ of the light is reflected by the mirrored surface, and the remaining $1 / 3^{\text {rd }}$ passes through the aperture to the lamp, and is assumed to be lost (though tests have indicated that a fraction may return to the integrator).

The remaining " $2 / 3$ rds" mentioned above is homogenized again on the way to the output end of the integrator, where red light is allowed to pass through the red segment of the wheel while reflected by the other segments, as described above. This process is repeated several times until all the light that entered the input aperture from the lamp is either transmitted to the modulator, absorbed, or scattered (Figure 4).

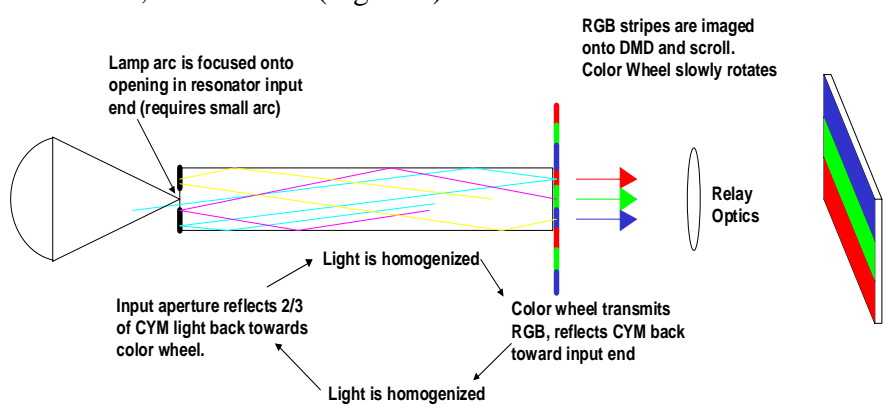

Figure 4. - The SCR recycling process

\subsection{Quantification of SCR Gain}

SCR gain is described as the increase in intensity of the RGB segments of the wheel, when imaged onto the modulator, over the case where no light recycling was present. When no recycling is present the scrolling system and a standard field-sequential system should have identical efficiency (provided the F/S wheel has equal size segments and no clear segment) since the duty cycle of a particular color on a given pixel of the modulator is exactly $1 / 3$. 
Also, when no recycling is present, the light that is not transmitted to the modulator through the relay optic is returned to the lamp and turned into heat. Simply put, when red is present on the modulator blue and green light must be wasted.

Consider an SCR system with an input aperture of 1/3 the area of the integrator. The light from the lamp which matches the red wavelengths is homogenized and reaches the wheel, and $1 / 3$ of the area of the integrator is covered by red-transmitting coatings, so $1 / 3^{\text {rd }}$ of the red light passes through the wheel to the modulator. The remaining $2 / 3$ of the red light returns to the input aperture/mirror, and $2 / 3$ of that light is returned to the output end where $1 / 3^{\text {rd }}$ of the red light is matched with the red coating and is transmitted. This process is repeated several times. The series $1 / 3\left[1+(2 / 3)^{2}+(2 / 3)^{4}+\ldots.\right]$ represents the fraction of light of a given color to eventually pass through the wheel. The series converges to $1 / 3[1.8]$, meaning that an increase in efficiency in the order of $80 \%$ can be realized.

Predictions of the expected efficiency gain can be more accurately predicted using the following formula

$$
G A I N={ }_{n=0}^{\infty}\left[\left(1-\frac{A_{1}}{A_{I N T}}\right)\left(1-\frac{A_{2}}{A_{I N T}}\right)\left(R_{1}\right)\left(R_{2}\right)\right]^{n}
$$

where $A_{I N T}$ is the area of the integrator, $A_{1}$ and $A_{2}$ are the areas of the input aperture and one of the color segments of the wheel, respectively, and $R_{1}$ and $R_{2}$ are the total reflectance (including absorption and scattering losses) of the input aperture mirror and color wheel segment, respectively. As can be seen, the recycling efficiency will increase as the ratio of the input aperture to the integrator cross-sectional area decreases. For instance, if the input aperture is $25 \%$ of the integrator area, the best-case theoretical efficiency boost is 2.0. Even though the efficiency boost can be increased by reducing the input aperture size, the reduced coupling of the arc into the smaller area may reduce overall efficiency of the projector.

\subsection{Thermodynamic Considerations}

Even though the predicted efficiency gain of 1.8 is much lower than the theoretical efficiency gain of 3.0 for a conventional scrolling system, practical application of the SCR optics decreases the advantage of conventional color scrolling. The SCR optical system has the same number of components as a conventional $\mathrm{DLP}^{\mathrm{TM}} 1$-chip optical system, namely, the wheel, rod integrator, and 3-5 element relay optics. On the other hand, a conventional scrolling-color system will require 3 color splitting dichroics, mirrors for positioning the 3 beams, optics for focusing each color onto the modulator, and optics for manipulation of the beams to cause the scrolling action. Each focusing step has the effect of increasing the etendue of the lamp due to irreversibility of optical aberrations, as well as providing opportunities for light to be lost due to absorption, scattering and misalignment. Also, properties of the scanning process can cause the color segments to change size as they travel over the modulator resulting in an overscan condition where areas outside the modulator array are illuminated. The combination of these losses brings a practical value for scrolling color efficiency gain down to a level on par with SCR predictions. It should also be noted that the mechanical complexity and volume required for conventional scrolling optics is much higher than for SCR illumination.

\subsection{Etendue Considerations}

The SCR light recapture process has the effect of increasing the etendue of the lamp arc by factor determined by the ratio of the rod exit area to the input aperture size, nominally 3.0. This agrees with earlier work [4] in that the lamp arc etendue must be decreased by a factor of three to achieve similar coupling efficiency as a non-scrolling system, since the arc must be focused onto an area of $1 / 3^{\text {rd }}$ the area of the modulator.

Figure 5 shows a graph of light collection for a UHP-type lamp (1.3mm arc gap) with an f/1 elliptical reflector as a function of aperture diameter (A1). This would correspond to the light that would be admitted into the SCR integrator for a given input aperture size. Also in the figure is a graph of equation (1) with the following assumptions:

Modulator: .7"XGA DLP

F/\# of illumination/projection: 2.4

Modulator etendue $=19.5 \mathrm{Str}-\mathrm{mm}^{2}$

$\mathrm{R}_{1}=\mathrm{R}_{2}=.95 ; \mathrm{A}_{2} / \mathrm{A}_{\mathrm{INT}}=1 / 3$

Figure 6 shows the product of the curves of Figure 5. Note that a value for $A_{1}$ can be selected corresponding to maximum overall projector efficiency. Note that the maximum efficiency occurs at an aperture diameter of $4.2 \mathrm{~mm}$, which is much larger than the $3.6 \mathrm{~mm}$ value, which would represent $1 / 3^{\text {rd }}$ of the integrator area. This is because the lamp arc etendue is much larger than would be desired for high projector efficiency.

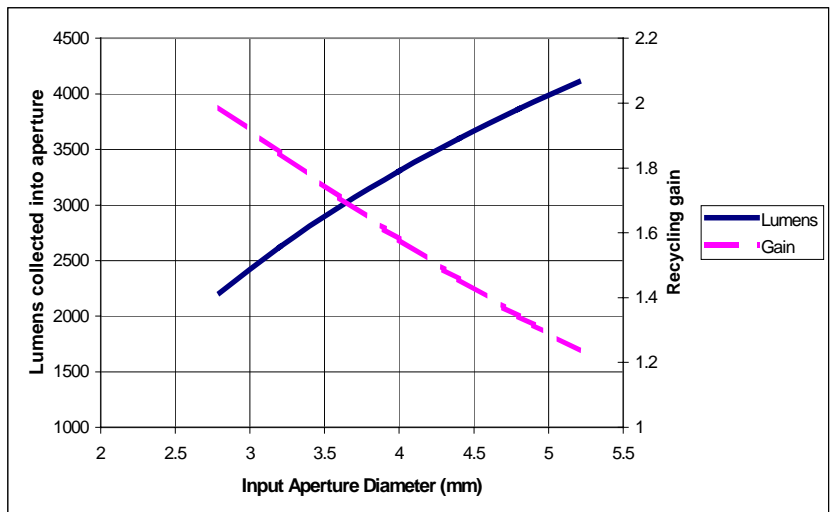

Figure 5 - Lamp Light Collection and Recycling Gain

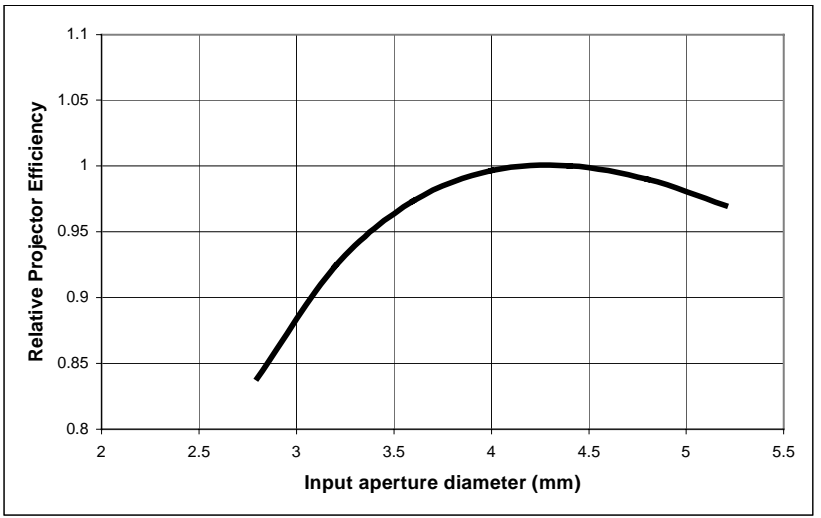

Figure 6 - Projector efficiency vs. Input aperture diameter 


\subsection{Prototype System}

During the summer of 2000 the Advanced Optics Technology Team of Texas Instruments' DLP ${ }^{\mathrm{TM}}$ Products began work on a Prototype SCR projection system.

\subsubsection{Prototype System Parameters:}

Modulator: 0.9" SXGA 13.8 micron pitch DMD

Illumination: $\mathrm{f} / 3$ telecentric

Integrator: $4.6 \times 6.2 \mathrm{~mm}$ solid BK7 with $3.6 \mathrm{~mm}$ aperture deposited on the input end.

Lamp: Osram 120W VHP with f/1.0 elliptical reflector

SCR Wheel: 126 segment spiral rotating at 660 RPM

Figure 7 shows the prototype optical system. A special formatter was designed to allow scrolling data to be displayed on the DMD device.

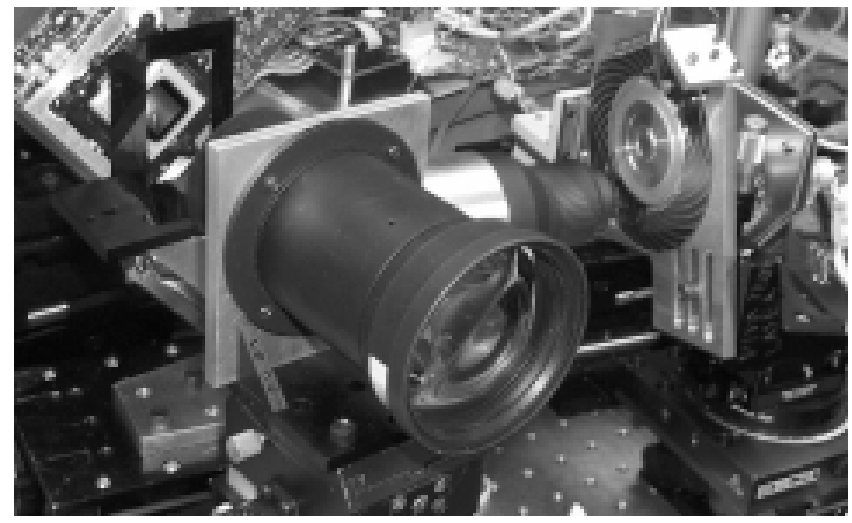

Figure 7 - SCR Prototype Optical System

\subsection{Predicted Performance}

Based on the color wheel filters used, the predicted "color wheel efficiency" of the spiral wheel was 0.342 . This is essentially the average photometric transmission of the wheel with an adjustment for "white boost", which is the use of the regions between the color bands, which are marginally cyan, yellow, and magenta light, to enhance the brightness of white content.

To calculate the predicted lumen output of the prototype SCR projector, one must multiply the following components of the light budget:

Lumens captured by the input aperture $=3300$

DMD Optical and Electronic efficiency $=0.68$

Optical efficiency including projection lens $=0.70$

SCR Gain Estimation = 1.67 (from gain formula with $5 \%$ loss at each end)
Color Wheel efficiency $=0.342$

The product of the above 5 numbers is 897 lumens, the predicted output of the SCR prototype projector.

\subsection{Measurements}

The SCR prototype was first measured in June 2000 in Plano, TX. ANSI brightness measurements of the image averaged 902 lumens, which was very close to the predicted value. Due to the non-flatness of the SCR wheel used, there was a variation of the spacing between the integrator output and the SCR wheel, which caused a noticeable pulsation in the picture. Clearly, this is an indication of the SCR effect. The lumenous efficiency of the prototype was 7.5 lumens/watt(lamp), which approaches 3-chip pSi projectors with a similar color gamut. Surprisingly, the ratio of screen lumens to input lumens (light entering the input aperture) was equal to that of 3-chip DLP ${ }^{\mathrm{TM}}$ projectors, implying that with a sufficiently small lamp arc or sufficiently high modulator etendue, the efficiency of 3-modulator projectors can be attained with this technique.

\section{Future Experiments and Discussion}

The Advanced Optics Technology team plans to continue experimentation with SCR and related optical components. The authors believe that 10 lumens/Watt(lamp) with SMPTE C colors can be optained, and that $>13$ lumens/Watt can be demonstrated with a single-DMD system with a less-saturated color gamut and a clear segment in the SCR wheel.

\section{Acknowledgements}

The authors would like to thank Dan Morgan, Don Doherty, Paul McFarland, Greg Hewlett, Claude Tew, and the employees of Texas Instruments who made the first SCR demonstration possible.

DLP and Digital Light Processing are trademarks of Texas Instruments, Incorporated.

\section{References}

[1] U.S. Patent 5,448,314 (Issued 09/05/1995) Texas Instruments, Inc.; Heimbuch, et. al.

[2] Florence, James M. and Yoder, Lars A, Display system architectures for digital micromirror device (DMD)-based projectors. Proc. SPIE Vol. 2650, p. 193-208, Projection Displays II(1996)

[3] U.S. Patent 5,410,370 (Issued 04/25/1995) Philips Corporation, Jannsen, Peter J.

[4] Stupp, Edward H., and Brennesholtz, Mattew S., Projection Displays (Wiley, 1999) pp. 229-230. 